\title{
A REVIEW ON ESTIMATION OF SERUM LDH AND URIC ACID IN HYPERTENSIVE VS NORMAL PREGNANT WOMAN AND ITS CORRELATION WITH MATERNAL OUTCOME in A TERITIARY CARE HOSPITAL.
}

\author{
Lincy Joseph ${ }^{1}$, Mathew George ${ }^{2}$, Anju Alex ${ }^{2 *}$ \\ ${ }^{1}$ Department of pharmaceutical chemistry, Pushpagiri college of Pharmacy, Thiruvalla- 689107, Kerala, India. \\ ${ }^{2}$ Department of pharmacology, Pushpagiri college of Pharmacy, Thiruvalla- 689107, Kerala, India.
}

\begin{abstract}
Hypertension is the most common medical problem encountered in pregnancy and remains an important cause of maternal and fetal morbidity and mortality. It complicates up to $15 \%$ of pregnancies. The review was intended to assess the prognostic significance of the value of serum LDH and used as a marker of preeclampsia. Higher serum LDH and uric acid level associated with increased incidence of maternal complications like abruption placenta, HELLP syndrome etc. There was an increase in maternal morbidity with increasing serum levels. To conclude high serum LDH and uric acid levels have significant association with severity of disease and maternal outcomes in patient of preeclampsia and can be considered as a prognostic tool from early trimester.
\end{abstract}

Key words: LDH, uric acid, hypertensive disorders.

\section{INTRODUCTION}

Pregnancy is a physiological state associated with many alterations in metabolic, biochemical, physiological, haematological and immunological process. If there are no complications, all these changes are reversible following a few days to a few months after delivery. Pregnancy can induce hypertension in women who were normotensive before pregnancy and aggravate hypertension in those who were hypertensive before pregnancy.

Hypertension during pregnancy is a major health problem. It is one of the leading causes of perinatal morbidity and mortality. It complicates about $5 \%$ to $10 \%$ of pregnancies in India. It is strongly associated with fetal growth retardation and prematurity .

Gestational hypertension is the new onset of hypertension with blood pressure more than or equal to $140 / 90 \mathrm{~mm}$ of $\mathrm{Hg}$ at mid pregnancy (greater than 20 weeks of gestation) without proteinuria. Preeclampsia is gestational hypertension pertension with proteinuria (at least $300 \mathrm{mg} / 24$ hours).Gesttional hypertension may progress to preeclampsia. Preeclampsia may be associated with certain complications in fetus like intrauterine growth restriction, prematurity and also may lead to death of fetus. Preeclampsia may be associated with certain complications in fetus like intrauterine growth restriction, prematurity,

*Corresponding author:

Email: anjualex42@gmail.com

http://dx.doi.org/10.20530/IJTA $32 \quad 35-37$

ISSN 2320-138X @ 2016 and may also lead to death of the fetus. Pulmonary Oedema, stroke, hepatic and renal failure may be develop in case of preeclamptic women. Pulmonary oedema and even stroke. Preeclampsia may progress to eclampsia, which is a form of hypertensive disorder of pregnancy with convulsion. Early screening of gestational hypertension and preeclampsia may reduce the maternal and fetal complications. Placental abruption, disseminated intravascular coagulation, intracranial haemorrhage, cardiovascular collapse hepatic failure and acute renal failure are the potentially lethal complications produced in preeclampsia. Lactate Dehydrogenase is an intracellular enzymes which is responsible for interconversion of pyruvate and lactate in the cells. LDH levels are greater inside the cells than in plasma. Preeclampsia is a multisystem disorder leads to lot of cellular death. Therefore serum LDH levels can be used to determine the extent of cellular death and thereby the severity of disease.LDH is mostly measured to evaluate the presence of tissue damage. The enzyme LDH is mainly found in many body tissues especially in liver, kidney, skeletal muscle, brain, blood cells, and lungs. Hence Serum LDH Levels can be further used as help in making decision, regarding the management strategies to improve the maternal and fetal outcome. Uric acid is an end product of purine metabolism. It is completely reabsorbed in the proximal convoluted tubules by carrier ted process and filtrated through the glomeruli . It is also actively secreted into the tubules. Total uric acid i.e. $85 \%$ is derived by tubular secretion. In preeclampsia hyperuricemia is found to be one of 
the earliest laboratory manifestation. Due to decreased renal urate excretion, there is elevated uric acid level in maternal blood and is frequently found in women with preeclampsia. Abnormal renal function, increased tissue breakdown acidosis and increased activity of enzyme xanthine oxidase dehydrogenase the several potential origins for uric acid in preeclampsia.. The cause of hyperureicemia in preeclampsia has been attributed to either a decreased excretion or to an increased production of uric acid. Decreased uric acid clearance, reflected by altered tubular function. Increased breakdown of purines in placenta as explanation of over production of uric acid. Soluble uric acid impairs nitric oxide generation in endothelial cells inducing endothelial dysfunction .Higher levels of Uric acid and Lactate Dehydrogenase (LDH) are a useful biochemical marker that reflects the severity of the occurrence of the complications of pre-eclampsia. The analysis of a combination of biochemical markers particularly markers related to vascular dysfunction such as increased uric acid, LDH, concentration may enrich the ability to predict and prevent $P E$ in near future.

\section{SERUM LDH IN PREECLAMPSIA}

Preeclampsia is a multisystem disorder. The organ dysfunction in severe preeclampsia caused by vascular endothelial damage including maternal liver, kidney, lungs, nervous system and coagulation system will lead to excessive LDH lekage and elevated levels in serum due to cellular dysfunction. In a study ${ }^{(4)} 100$ pregnant women were taken,50 as control with normal pregnant women and 50 preeclampsia. The mean serum LDH was significantly higher in preeclampsia patients. The higher levels of serum LDH can be a useful marker to identify the occurrence of complications of preeclampsia in early pregnancy which may reduce the risk of occurence of disease. $\mathrm{LDH}$ is an intracellular cytoplasmic enzyme ${ }^{(6)}$. LDH enzymes are ubiquitous to all the major organ systems.eg kidney ,muscle, leukocytes erythrocytes.LDH isoenzymes can be measured and can be further used as help in making decision regarding the management strategies to improve maternal and fetal outcome.LDH levels have significant association with severity of disease in patients of preeclampsia and ecclampsia and considered as a supportive prognostic tool from early third trimester.

\section{SERUM URIC ACID IN PREECLAMPSIA}

Uric acid is an end product of purine metabolism and filterated through glomeruli and almost completely reabsorbed in proximal convoluted tubules $^{(3)}$. Hyperuricemia is found to be one of the earliest laboratory manifestations of preeclampsia. Its increased levels suggest serious impending damage to kidney functions. A significant increase in serum uric acid levels was observed in gestational hypertension and preeclampsia compared to normal pregnancy ${ }^{(2)}$. A close monitoring of serum uric acid level during the period of pregnancy may help in early detection and

Intervention of gestational hypertension and preeclampsia in preventing complications that may arise due to disease.

\section{SERUM LDH AND URIC ACID IN PREECLAMPSIA}

Comparing the serum LDH and uric acid in hypertensive and normotensive pregnant woman ${ }^{(1)} \mathrm{A}$ significant increase in serum LDH and uric acid level are found in women with hypertension than normal pregnant woman. Estimation of serum uric acid is as important as proteinuria in identifying the risk of renal involvement. These findings indicate that increased levels of these parameters are seen as the disease severity increases. Serum LDH and uric acid are reliable and inexpensive markers to predict severity.

\section{SERUM LDH , URIC ACID AND ITS COORELATION WITH MATERNAL OUTCOME}

Serum levels of LDH, uric acid, AST, ALT was statistically significant with maternal deaths in comparison to mothers who survived (7). Preeclampsia produces potentially lethal complications including placental abruption, hepatic failure, acute

Renal failure and cardiovascular collapse. The analysis of a combination of biomarkers particularly markers related to vascular dysfunction such as LDH and uric aid may enrich the ability to predict and prevent preeclampsia in near future. The women with preeclampsia ,elevated uric acid concentration indicate pregnancies at increased risk for preterm delivery compared with women with preeclampsia in the absence of hyperuricemia ${ }^{(5)}$.The pregnant women with preeclampsia associated with elevated serum uric acid levels were at greater risk for complications and adverse pregnancy outcome. In a study the subjects are divided according to serum LDH levels ${ }^{(6)}$.

When the LDH levels were $<600$ there were two cases of postpartum haemorrhage and one case of abruption placenta. In group were LDH levels were $600-800$, one case of pulmonary 
oedema and postpartum haemorrhage were observed. There was statistically significant increase in maternal complications with increasing LDH levels. High LDH levels have significant association with maternal outcome.

\section{CONCLUSION}

Preeclampsia is a multisystem disorder, characterized by vascular endothelial dysfunction. Various studies found that a statistically significant difference between serum uric acid, LDH with maternal death in PIH cases. So higher levels of these parameters can be very useful markers to identify the occurrence of the complications of preeclampsia in early pregnancy and help in the management of the women with preeclampsia.

Results of the review indicated association of elevated serum uric acid level with preeclampsia which could be used as a biochemical indicator of preeclampsia in pregnant women. Elevated levels of lactic dehydrogenase, indicative the cellular damage and dysfunction can be used as a biochemical marker because it reflects the severity of the disease. Standard antenatal follow up should be carried out for the early detection and prevention of PIH. A close monitoring of serum uric acid level and $\mathrm{LDH}$ during the period of pregnancy may help in the early detection and intervention of gestational hypertension and preeclampsia and thereby help in preventing the maternal and fetal complications due to preeclampsia. The women with preeclampsia

\section{REFERENCES}

1. Gandhi M, Chavda R, Saini HB. Comparative study of serum LDH and uric acid in hypertensive versus normotensive pregnant woman. Int Jour of Biomed Res. 2015 Jan 30;6(1):25. Available from: http://dx.doi.org/10.7439/ijbr.v6i1.1522.
2. Julie Sarmah. A Comparative Study of Serum Uric Acid in Gestational Hypertension, Preeclampsia and Normal Pregnancy. IOSR Journal of Dental and Medical Sciences (IOSR-JDMS). 2015 Aug ;14( 8) :46.

3. Amit D. Sonagra, Dattatreya.K, Jayaprakash Murthy D.S. Serum LDH, ALP and Uric acid in hypertensive disorders of pregnancy. International Journal of Pharmacy and Biological Sciences. 2012 July-Sept; 2(3): 201-209.

4. Rubina Aziz, Tabassum Mahboob. Relation between preeclampsia and cardiac enzymes. Arya Atherosclerosis journal. 2008; 4(1):29-38.

5. Kamath R, Nayak R, Shantharam M. Serum Uric acid level in preeclampsia and its correlation to maternal and fetal outcome. Int Jour of Biomed Res. 2014 Jan 30;5(1):22. Available from: http://dx.doi.org/10.7439/ijbr.v5i1.457.

6. Y. Umasatyasri, I.Vani, P.Shamita.Role of LDH (Lactate dehydrogenase) in preeclampsia eclampsia as a prognostic marker: An observational study. International Archives of Integrated Medicine .2015 September;2 (9):88-93.

7. L A, L M, A S, N H, S V, P J. Maternal outcome in relation to Biochemical parameters in Hypertensive disorders in Pregnancy. IOSR Journal of Dental and Medical Sciences. 2014;13(2):18-22. Available from: http://dx.doi.org/10.9790/0853-13221822.

8. Julie Sarmah. Evaluation of Serum Aspartate Aminotransferase (AST), Alanine Aminotransferase (ALT), Alkaline Phosphatase (ALP), Lactate Dehydrogenase (LDH) and Uric Acid In Preeclampsia. IOSR Journal of Dental and Medical Sciences (IOSR-JDMS). 2015 June; 14(6): 10-12. 